Research Article

\title{
Cyclic Voltammetry and Oxidation Rate Studies of Ferrous Gluconate Complex Solutions for Preparation of Chitosan-Tripolyphosphate Microparticles
}

\author{
Noer Abyor Handayani (D, Elsa Anisa Krisanti, Sutrasno Kartohardjono (D), \\ and Kamarza Mulia \\ Department of Chemical Engineering, Faculty of Engineering, Universitas Indonesia, Kampus UI Depok, Depok 16424, Indonesia \\ Correspondence should be addressed to Kamarza Mulia; kmulia@che.ui.ac.id
}

Received 26 August 2019; Accepted 27 January 2020; Published 26 February 2020

Academic Editor: Maolin Guo

Copyright (c) 2020 Noer Abyor Handayani et al. This is an open access article distributed under the Creative Commons Attribution License, which permits unrestricted use, distribution, and reproduction in any medium, provided the original work is properly cited.

\begin{abstract}
A proper understanding of the properties of iron could increase the effectiveness of programmes for alleviating iron deficiency. Recently, encapsulation has been considered an appropriate method for protecting iron from injurious reactions. However, several events may occur during encapsulation processes, including changes in the iron's oxidation state. Oxidation of ferrous iron is not desirable since the intestines can only absorb iron in the ferrous form. In this study, a cyclic voltammetry method was applied to investigate the likelihood of ferrous gluconate oxidation for the preparation of chitosan-tripolyphosphate microparticles. Then, the electrochemical properties of ferrous gluconate were confirmed experimentally. The oxidation rate of ferrous gluconate is also discussed in this paper. All the experimental solutions were formulated in detail to produce conditions similar to those of microparticle production. Cyclic voltammetry analysis was conducted using a configuration of three electrodes connected to an electrochemical analyser. Graphite, platinum wire, and $\mathrm{Ag} / \mathrm{AgCl}$ were employed as the auxiliary, working, and reference electrodes, respectively. The cyclic voltammetry results show that the observed potential for each anodic peak shifted negatively in the presence of chitosan and sodium tripolyphosphate. Moreover, the rate of ferrous oxidation tended to increase during 75 min of experiments due to the presence of chitosan and sodium tripolyphosphate. These behaviours indicate the transformation of ferrous iron to ferric iron during iron microparticle preparation. Furthermore, these findings suggest that spray drying is a preferable method to minimise the oxidation reaction.
\end{abstract}

\section{Introduction}

Currently, iron deficiency (ID) is an important issue in public nutrition, affecting $20 \%$ of the world's population $[1,2]$. ID can disrupt oxygen transportation and enzyme reactions due to the depletion of erythrocyte and haemoglobin [3-5]. These effects may also interfere with metabolic pathways bringing about negative consequences in terms of growth, cognitive capability, mental development, immune function, and reproductive performance, with children under 5 years old and women of child bearing age being the most affected subjects [6-10]. In women, ID can form a vicious circle, if the amount of iron does not meet the body's needs starting from infancy, through adolescence, and worsening in pregnancy, thereby increasing the risk of giving birth to infants with low iron sources $[1,4,5,11,12]$. The Indonesian government has implemented two programmes for ID prevention through iron supplementation and fortification $[9,10]$. Indeed, since the 1970s, women have been advised to consume 90 tablets of iron/folic acid supplements during pregnancy [6]. Moreover, the iron fortification of flour has been implemented since 1998 . However, the prevalence of ID in Indonesia remains higher than $15 \%$ due to the following factors: (i) low dietary intake of iron (less than $20 \mathrm{mg} /$ day), (ii) poor bioavailability, and (iii) chronic blood loss by infection (in certain regions) $[4,7,13]$.

Fortification and supplementation programmes may not be optimal because of the reduced availability of ferrous iron due to the presence of inhibitors (phytic acid, tannins, and 
casein), competitors (calcium and zinc), or oxidising compounds [5, 14-19]. The interaction of iron with other dietary compounds can lead to the formation of unavailable iron components in the gastrointestinal tract, thereby decreasing bioavailability [17]. Given these properties, encapsulation is considered a suitable method to protect iron from oxidation and other injurious reactions, by forming an impermeable barrier $[8,14,20]$.

One carbohydrate polymer candidate for encapsulation of protein and other active compounds is chitosan (Ch). It has excellent mechanical, biocompatible, biodegradable, nontoxic, mucoadhesive, and antimicrobial properties [20-22]. Ch entraps compounds by forming covalent or ionic cross-linked networks with glyoxal, glutaraldehyde, and tripolyphosphate (Tp) $[21,23]$. In this study, ferrous gluconate $(\mathrm{FeG})$ was used as an iron source because of its high bioavailability and stability under oxidation compared with other ionic ferrous salts $[6,15,19]$. The standard oxidation potential of $\mathrm{Fe}^{2+}$ at $25^{\circ} \mathrm{C}$ $(298 \mathrm{~K})$ is $-0.77 \mathrm{~V}$. The challenge in iron microencapsulation is dealing with its instability with respect to its environment. Several issues may occur during the preparation of iron microparticles, including changes of the iron state due to oxidation. Previous work succeeded in producing FeG microparticles using $\mathrm{Ch}$, acetic acid (Ac), and Tp as the encapsulating material, solvent, and cross-linking agent, respectively [15]. That study showed that the amount of encapsulated ferrous iron was less than the total iron. This might be because some of the ferrous iron was transformed into ferric compounds during microparticles preparation. Oxidation of ferrous iron into ferric ones is not desirable, since the intestines can only absorb iron in its ferrous form $[15,17,18]$. Hence, further investigation of the redox behaviour of iron is urgently required.

Cyclic voltammetry (CV) is commonly applied method to observe redox potential behaviour by measuring the current generated from the electron transfer between an electrode and an electrolyte solution as a function of changes in potential. This experiment utilises a cell equipped with three electrodes: the reference, working, and auxiliary electrodes [24-29]. CV is frequently used in applied electrochemistry, analytical electrochemistry, and surface coating $[24,25,28-30]$. In contrast, there is a lack of information regarding its application in the food and health fields. Previous work examined the redox properties of ferrous and ferric upon addition of nicotine, and the correlation with neurodegenerative disease [26]. The ability of nicotine to form a complex reaction with free iron was proved by the change from reversible to quasi-reversible performances, as well as a reduced rate of electron transfer and the apparent rate constant $\left(k_{s}\right)$; thus, it successfully reduced iron reactivity [26]. Another study investigated the structure antioxidant activity relationship (SAR) of flavonoids towards different free radicals by ferric reducing antioxidant power (FRAP) with slight modification using 96-well microplates [27]. Their oxidation potentials were analysed by the CV method and found to be in good agreement with the FRAP results [27].

Currently, there is insufficient information about the redox activity of FeG complex solution for preparation of $\mathrm{Ch}$-Tp microparticle. Therefore, the aim of this study was to investigate the likelihood of $\mathrm{FeG}$ oxidation using the $\mathrm{CV}$ electrochemical method. However, the electrochemical properties of $\mathrm{FeG}$ in the presence of $\mathrm{Ac}, \mathrm{Ch}$, and $\mathrm{Tp}$ should be confirmed by laboratory experiments. Hence, the ferrous oxidation rate of $\mathrm{FeG}$ was also discussed in this paper.

\section{Materials and Methods}

2.1. Materials. Ferrous gluconate was purchased from Dalian Chemical \& Export Group Co. Ltd, China. Chitosan was supplied from CV. ChiMultiguna Cirebon, Indonesia. Acetic acid (99.99\%), sodium tripolyphosphate, hydroxylamine hydrochloride, sodium acetate, and 1,10-phenanthroline were provided by Merck. All solutions were prepared with aquadest from PT. Brataco, Indonesia. The molecular structure of each material is described in Figure 1.

2.2. Preparation of Solutions. Four electrolyte solutions were prepared to investigate the effect of each compound normally used in the FeG microparticles preparation using $\mathrm{Ch}$ and $\mathrm{Tp}$ as encapsulating material and cross-linking agent, respectively. The electrochemical behaviour of ferrous compounds in all formulations was observed using the $\mathrm{CV}$ method.

2.2.1. Ferrous Gluconate Solution $(\mathrm{FeG})$. FeG solutions of varying concentrations $(5,10$, and $15 \mathrm{mM})$ were prepared by dissolving FeG in oxygen-free aquadest. These solutions are referred to as the blank samples.

\subsubsection{Ferrous Gluconate-Acetic Acid (FeG-Ac) Solution.} This formulation was similar to the FeG solution, except that the solvent was replaced with $2.5 \%(\mathrm{v} / \mathrm{v})$ Ac. Various concentrations of $\mathrm{FeG}(5,10$, and $15 \mathrm{mM})$ were investigated.

\subsubsection{Ferrous Gluconate-Acetic Acid-Chitosan (FeG-Ac-Ch)} Solution. FeG-Ac-Ch solution was prepared by dissolving FeG in $2.5 \%(\mathrm{v} / \mathrm{v})$ of Ac. Afterwards, different concentrations of $\mathrm{Ch}(0.25,0.50$, and $0.75 \% \mathrm{w} / \mathrm{v})$ were prepared. The density and viscosity of this solution were also analysed due to its physical changes.

\subsubsection{Ferrous Gluconate-Acetic Acid-Chitosan-Tripolyphos-} phate (FeG-Ac-Ch-Tp) Solution. FeG-Ac-Ch-Tp solution was made by dissolving FeG in $2.5 \%$ (v/v) Ac. The Ch was then added and mixed until the solution was homogeneous. Next, Tp solution in various concentrations $(0.25,0.5$, and $0.75 \% \mathrm{w} / \mathrm{v}$ ) was added using a syringe to the FeG-Ac-Ch solution. The amount of $\mathrm{Tp}$ solution was $5 \%$ of the total volume.

\subsection{Electrochemical Analysis: CV Method. A cyclic voltam-} mogram was acquired using a configuration of three electrodes connected to an electrochemical analyser (Autolab Metrohm Potentiostat, Galvanostat PGSTAT 302 N). Platinum wire, graphite, and $\mathrm{Ag} / \mathrm{AgCl}$ (with an electrolyte concentration of $3 \mathrm{M} \mathrm{KCl}$ ) were used as the working, auxiliary, and reference 
<smiles>O=C(O[I+]OC(=O)[C@@H](O)[C@H](O)[C@H](O)CO)[C@@H](O)[C@@H](O)[C@H](O)[C@H](O)[C@H](O)CO</smiles>

(a)<smiles>CC(=O)O</smiles>

(b)<smiles>NC1C(OC2C(CO)OC(OC3C(CO)OC(O)C(N)C3O)C(N)C2O)OC(CO)C(O)C1O</smiles>

(c)<smiles>[N]OP(=O)(O[Na])OP(=O)(O[Na])O[Na]</smiles>

(d)

Figure 1: Molecular structure of (a) FeG, (b) Ac, (c) Ch, and (d) Tp.

electrodes, respectively. Furthermore, the solvent used was kept free of oxygen by degassing for 5 minutes prior to each experiment using an Elmasonic $\mathrm{S} 40 \mathrm{H}$ sonicator. Each solution was mixed at room temperature in a closed system to avoid further oxygen interference.

2.4. $p H$ Measurement. The acid-base behaviours of all investigated solutions were determined using a Hanna digital $\mathrm{pH}$ meter at $25^{\circ} \mathrm{C}$. Both standard $\mathrm{pH}$ buffers (4.0 and 6.8) were utilised for calibration. The $\mathrm{pH}$ measurement was carried out prior to electrochemical analysis and again after the analysis was completed.

2.5. Density Measurement. The pycnometer method was used to determine the density of the FeG-Ac-Ch solution. The pycnometer was purchased from PT. Iwaki Glass Indonesia and calibrated using the ISO 4787:1984 and ISO 3507:1999 methods. The measurements were carried out three times at $25^{\circ} \mathrm{C}$ for each sample, and then the final density values were averaged.

2.6. Viscosity Measurement. The viscosity of the FeG-Ac-Ch solution was determined following the standard ASTM D445-17 method.

2.7. Ferrous and Total Iron Measurement. The amounts of ferrous and total iron in the aqueous solution were determined using the phenanthroline method, as described in a previous study [14]. In this method, 1,10-phenanthroline, sodium acetate, and hydroxylamine hydrochloride are utilised as the complexing agent, $\mathrm{pH}$ buffer, and reducing agent, respectively. The colour absorbance was analysed using a Thermo Scientific Spectrophotometer (GENESYS 10S) at a wavelength of $510 \mathrm{~nm}$.

\section{Results and Discussion}

3.1. Determination of the Proper Conditions for Cyclic Voltammetry Analysis. In this study, various scan rates were applied, and different concentrations of $\mathrm{FeG}, \mathrm{Ch}$, and $\mathrm{Tp}$ were used to determine the appropriate conditions for $\mathrm{CV}$ analysis, so that a clear cyclic voltammogram could be obtained. Figure 2 depicts the reduction oxidation activity of each proposed solution at various scan rates $(v)$ in the potential $(V)$ range of -0.80 to $0.0 \mathrm{~V}$. In general, the cathodic peaks were not distinct in the four formulations, while anodic peaks were slightly observable. The recorded current peak $\left(i_{p}\right)$ decreased as slower $v$ was applied, which is in good agreement with previous reports [24, 26, 28, 31]. This result could be explained by considering the size of diffusion layer around the electrode and the time required to observe it. Since a slower scan rate was applied, a cyclic voltammogram would take longer to record and there was greater growth of the diffusion layer compared with a faster rate. As a result, the electron flux to the electrode surface was smaller; hence, a lower peak current was obtained, as the current is proportional to the flux towards the electrode. This is shown in equation (1), where $C$ is the concentration of FeG complex solution, $D$ is the diffusion coefficient, $A$ is the surface area of the working electrode, and $\alpha$ is the transfer coefficient [32]. However, the anodic potentials also became more positive. Furthermore, a higher and clear current was obtained using the higher $v$; thus a value for $v$ of $0.2 \mathrm{~V} / \mathrm{s}$ was applied in later investigations.

$$
i_{p}=2.99 \times 10^{5} v^{1 / 2} C D^{1 / 2} A \alpha^{1 / 2} .
$$

Figure 3 shows the cyclic voltammograms for FeG and FeG-Ac solutions at various FeG concentrations (5, 10, and $15 \mathrm{mM}$ ) at $v=0.2 \mathrm{~V} / \mathrm{s}$, in the $V$ range of -1.0 to $0 \mathrm{~V}$. In general, a higher current was recorded as the FeG concentration increased, due to the increase transfer of electron towards the electrode. These results are similar to those of the previous reports $[24,26,28]$. Because FeG concentrations of $10 \mathrm{mM}$ and $15 \mathrm{mM}$ did not represent the significant recorded current (Figure 2(b)), a concentration of $10 \mathrm{mM}$ was used for the next set of observations.

The electrochemical behaviour of FeG was also investigated using different $\mathrm{Ch}$ and $\mathrm{Tp}$ concentration, as shown in Figures 4(a) and 4(b), respectively. All experiments were conducted at $v=0.2 \mathrm{~V} / \mathrm{s}$ in a $V$ range of -0.8 to $0 \mathrm{~V}$. Figure 4 shows that an increase in the $\mathrm{Ch}$ and $\mathrm{Tp}$ concentrations did not significantly alter the observed current $(i)$, which might 


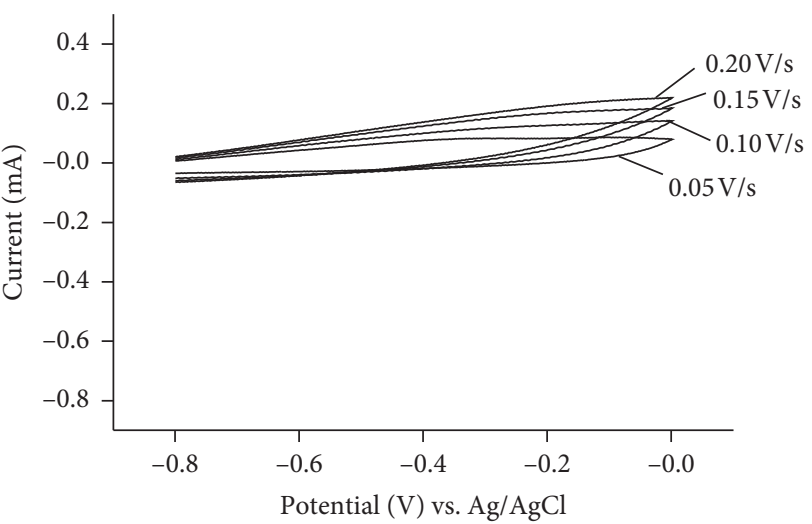

(a)

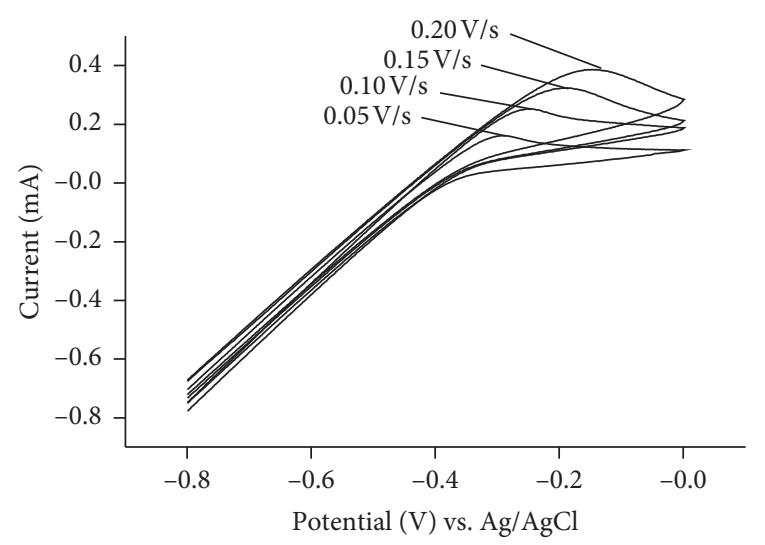

(c)

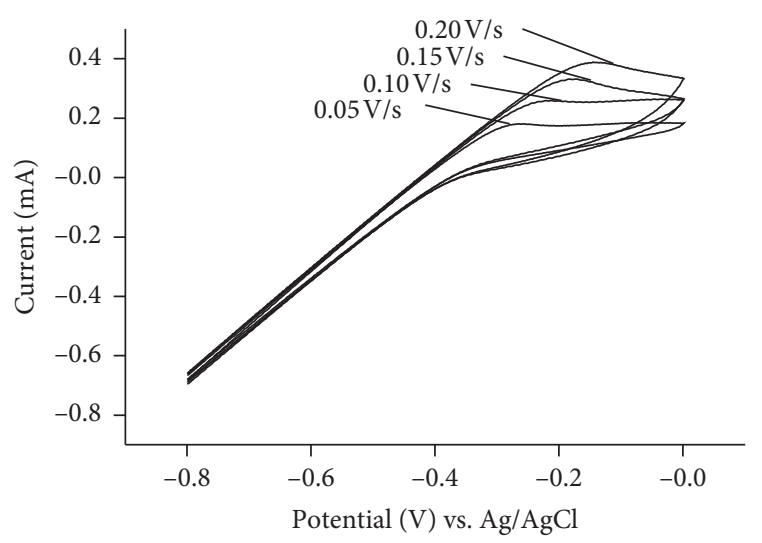

(b)

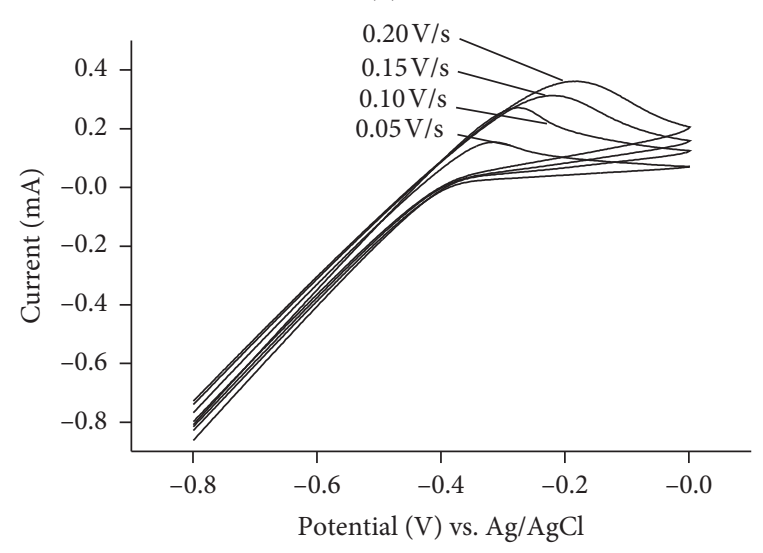

(d)

Figure 2: Cyclic voltammograms of (a) FeG $10 \mathrm{mM}$ solution, (b) FeG $10 \mathrm{mM}$-Ac 2.5\% solution, (c) FeG $10 \mathrm{mM}$-Ac 2.5\%-Ch 0.5\% solution, and (d) FeG $10 \mathrm{mM}$-Ac $2.5 \%$-Ch $0.5 \%$-Tp $0.25 \%$ solution, at $v$ values of $0.5,0.10,0.15$, and $0.20 \mathrm{~V} / \mathrm{s}$.

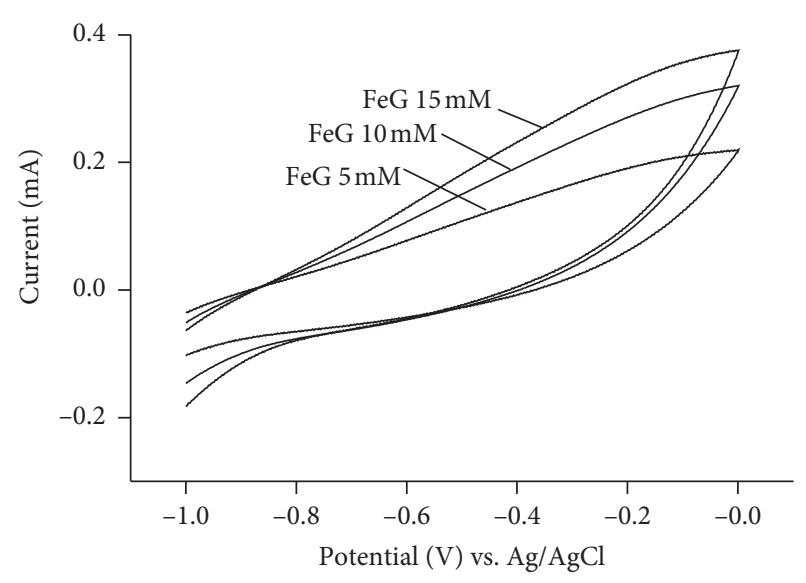

(a)

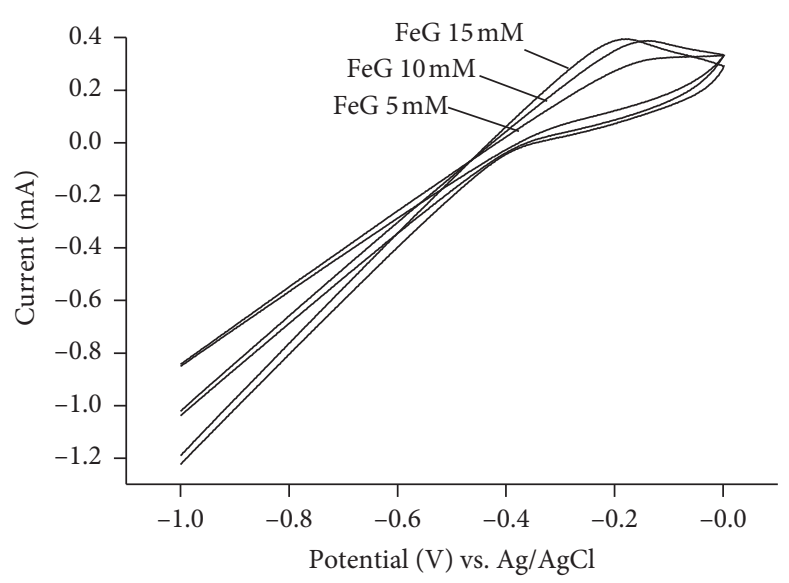

(b)

Figure 3: Cyclic voltammograms of (a) FeG solution and (b) FeG-Ac 2.5\% solution with varying concentrations of FeG (5, 10, and $15 \mathrm{mM}$ ) at $v=0.20 \mathrm{~V} / \mathrm{s}$.

be caused by a fixed amount of oxidised component. However, the value of $V$ for each $i_{p}$ became more negative with the increasing of $\mathrm{Ch}$ concentration. Given these observations, a higher concentration of chitosan induced ferrous compounds being more susceptible to entering ferric compounds. The density and viscosity were also analysed, due to the physical changes following $\mathrm{Ch}$ addition. A higher $\mathrm{Ch}$ concentration would increase density and viscosity values of FeG-Ac solution (Table 1). The high viscosity may have disrupted the electron transfer towards the electrode, 


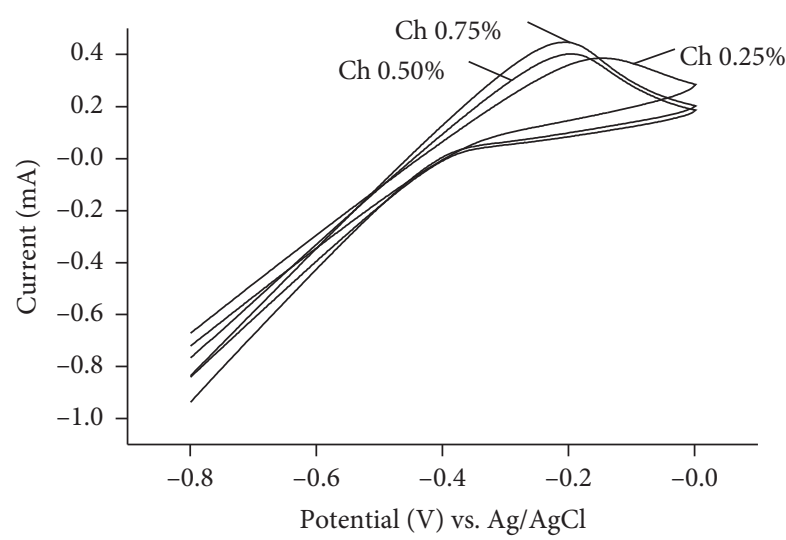

(a)

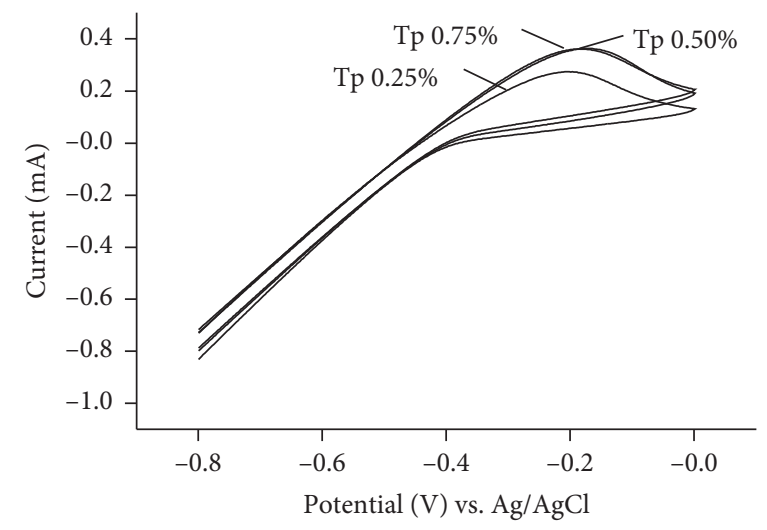

(b)

Figure 4: Cyclic voltammograms of solution containing (a) FeG $10 \mathrm{mM}-\mathrm{Ac} 2.5 \%$ at varying Ch concentrations and (b) FeG $10 \mathrm{mM}-\mathrm{Ac}$ $2.5 \%-\mathrm{Ch} 0.50 \%$ at various Tp concentrations, $v=0.20 \mathrm{~V} / \mathrm{s}$.

TABle 1: Density and viscosity of FeG-Ac solution and FeG-Ac-Ch solution.

\begin{tabular}{|c|c|c|c|c|c|c|c|}
\hline \multirow{3}{*}{ FeG-Ac solution } & \multicolumn{4}{|c|}{ Density at $25^{\circ} \mathrm{C}(\mathrm{g} / \mathrm{ml})$} & \multicolumn{3}{|c|}{ Viscosity at $25^{\circ} \mathrm{C}$ (Cst) } \\
\hline & \multicolumn{3}{|c|}{$\begin{array}{l}\text { FeG-Ac-Ch solution at various } \mathrm{Ch} \\
\text { concentrations }\end{array}$} & FeG-Ac solution & \multicolumn{3}{|c|}{$\begin{array}{l}\text { FeG-Ac-Ch solution at various } \mathrm{Ch} \\
\text { concentrations }\end{array}$} \\
\hline & $0.25 \%$ & $0.50 \%$ & $0.75 \%$ & & $0.25 \%$ & $0.50 \%$ & $0.75 \%$ \\
\hline 1.026 & 1.03 & 1.03 & 1.04 & 1.00 & 4.50 & 10.15 & 20.22 \\
\hline
\end{tabular}

thus interrupting the current recording. However, the cyclic voltammogram also shows that the addition of $0.5 \% \mathrm{Ch}$ led to obvious oxidation property as demonstrated by the negative shift of the anodic potential compared with the addition of $0.25 \% \mathrm{Ch}$. Furthermore, the addition of $0.75 \%$ $\mathrm{Ch}$ did not shift the anodic potential significantly; thus a $\mathrm{Ch}$ concentration of $0.5 \%$ was used for further experiments, so a clear potential alteration could be recorded.

Figure 4(b) shows that the increase in the Tp concentration changed the oxidation potentials from $-0.20 \mathrm{~V}$ to $-0.17 \mathrm{~V}$ and $-0.19 \mathrm{~V}$ indicating that $\mathrm{FeG}$ was more prone to oxidise. Also, the increase of Tp concentration from $0.25 \%$ to $0.50 \%$ and $0.75 \%$ did not significantly alter the anodic potential; thus a $\mathrm{Tp}$ concentration of $0.25 \%$ was applied for further investigation, so an obvious potential shift would be observed.

\subsection{Electrochemical Properties of FeG Complex Solutions for} Preparation of Chitosan-Tripolyphosphate Microparticles. The experiments were carried out using FeG $10 \mathrm{mM}$, Ac $2.5 \%$, Ch $0.5 \%$, and $\mathrm{Tp} 0.25 \%$ at $v=0.2 \mathrm{~V} / \mathrm{s}$, in the $V$ range of -0.8 to $0 \mathrm{~V}$. All the solutions were formulated to simulate the actual solution used in iron microparticle production. The electrochemical behaviours of each solution are represented in Figure 5. The values for the anodic current, anodic potential, and $\mathrm{pH}$ of each solution are also given in Table 2.

In general, the observed currents (i) became more positive and more negative with the presence of $\mathrm{Ac}, \mathrm{Ch}$, and Tp. The cyclic voltammogram for the FeG solution showed that there were no distinct oxidation and reduction peak. However, currents around zero were recorded during the

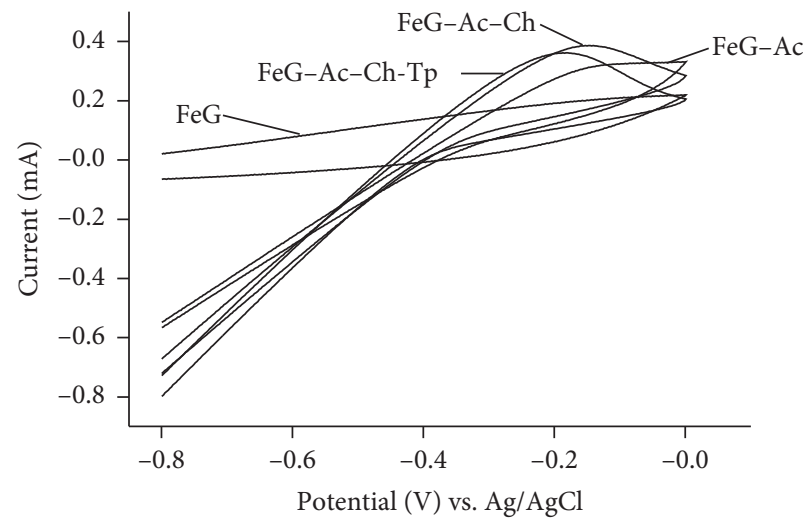

Figure 5: Cyclic voltammogram of complex solutions containing FeG $10 \mathrm{mM}$, Ac $2.5 \%$, Ch $0.5 \%$, and Tp $0.25 \%$ during iron microparticle preparation.

scanning process in the area of both oxidation and reduction; thus, a slight change of potential could deliver both anodic and cathodic currents.

In this study, Ac was used to dissolve $\mathrm{Ch}$, and $\mathrm{FeG}$ was then added to the solution as encapsulated material. The stability of FeG in Ac should be investigated; hence, the first step was to analyse the FeG-Ac solution. The presence of Ac led to a decrease in the $\mathrm{pH}$ of the FeG-Ac solution. This may have been due to the partial dissociation of Ac to produce protons $\left(\mathrm{H}^{+}\right)$in the aqueous phase, thereby increasing the acidity (Figure 1). Ac could also inhibit the polymerisation of iron in solution, thereby keeping it in its reactive state in the acidic medium [33, 34]. 
TABle 2: Values of anodic peak, and pH of complex solutions containing FeG $10 \mathrm{mM}$, Ac $2.5 \%$, Ch $0.50 \%$, and $\mathrm{Tp} 0.25 \%$ at $v=0.20 \mathrm{~V} / \mathrm{s}$.

\begin{tabular}{lcccc}
\hline \multicolumn{2}{c}{ Anodic peak } & pH prior to analysis & pH after analysis \\
& Current $(\mathrm{mA})$ & Potential $(\mathrm{V})$ & 4.1 & 4.0 \\
FeG & --- was not observable--- & 3.3 & 3.3 \\
FeG-Ac & 0.388 & -0.145 & 3.4 & 3.4 \\
FeG-Ac-Ch & 0.386 & -0.148 & 3.6 & 3.6 \\
FeG-Ac-Ch-Tp & 0.362 & -0.204 & & \\
\hline
\end{tabular}

The $\mathrm{Ch}$ in the FeG-Ac-Ch solution served as the encapsulating agent. Because of its rigid crystalline structure, it is only soluble in an acidic medium, such as Ac [20,35]. The oxidation peak of $\mathrm{FeG}-\mathrm{Ac}-\mathrm{Ch}$ increased from 0.33 to $0.39 \mathrm{~mA}$, while $V$ became more negative, falling from -0.14 to $-0.15 \mathrm{~V}$ (Table 1). This indicates that the ferrous iron was more susceptible to oxidation compared with the FeG-Ac solution. The presence of $\mathrm{Ch}$ also led to a slightly more alkaline $\mathrm{pH}$ (Table 1). Ch is a weak base polymer, since it has a large number of amino groups (Figure 1), and therefore increased the alkalinity of the FeG-Ac-Ch solution [21].

The chitosan application for oral delivery is closely related to controlled release systems; thus, the presence of Tp as a cross-linking agent is important $[35,36]$. The oxidation peak of the FeG-Ac-Ch-Tp solution became more negative, dropping from 0.39 to $0.36 \mathrm{~mA}$ in the presence of $0.25 \%$ (w/ v) Tp. However, the $V$ also became more negative, which indicates that it was easier to transform ferrous iron in the FeG-Ac-Ch-Tp solution into ferric iron in comparison with the FeG-Ac and FeG-Ac-Ch solution. The addition of Tp also produced a more alkaline $\mathrm{pH}$ in the FeG-Ac-Ch-Tp solution in comparison with the FeG-Ac-Ch solution. This occurred because of the presence of $\mathrm{Tp}$ anion which attracts $\mathrm{H}^{+}$ions.

The $\mathrm{pH}$ of each solution was affected only by the presence of $\mathrm{Ac}, \mathrm{Ch}$, and $\mathrm{Tp}$ (Table 1). The $\mathrm{pH}$ value of each sample measured following the experiment did not significantly differ from the $\mathrm{pH}$ recorded prior to the experiment. In accordance with the cyclic voltammogram results, all four solutions showed a tendency of ferrous state transformation during iron microparticle preparation.

3.3. FeG Oxidation Rate in Aqueous Solutions. Four solutions were formulated to study the likelihood of FeG oxidation using CV method. However, the electrochemical properties of $\mathrm{FeG}$ in the presence of $\mathrm{Ac}, \mathrm{Ch}$, and $\mathrm{Tp}$ needed to be confirmed experimentally. Hence, the concentration of ferrous iron as a function of time was investigated for $75 \mathrm{~min}$ (the average time for iron microparticle preparation), as depicted in Figure 6. The observed solution was prepared using $10 \mathrm{mM}$ of $\mathrm{FeG}, 0.5 \% \mathrm{Ch}$, and $0.25 \% \mathrm{Tp}$. A sample was collected every $15 \mathrm{~min}$, for ferrous determination using the phenanthroline method.

Figure 6 shows that $30 \%$ of the ferrous iron in the FeG solution was lost in the first $15 \mathrm{~min}$ of the experiment. This result supports previous work reporting that about $16.6 \%$ of the ferrous iron was lost from FeG solution after storage in a closed container for a day [37]. In contrast, only $5 \%$ of the ferrous iron in the FeG-Ac solutions was lost at the first $15 \mathrm{~min}$ and it stabilised in the last $60 \mathrm{~min}$ of the experiment.

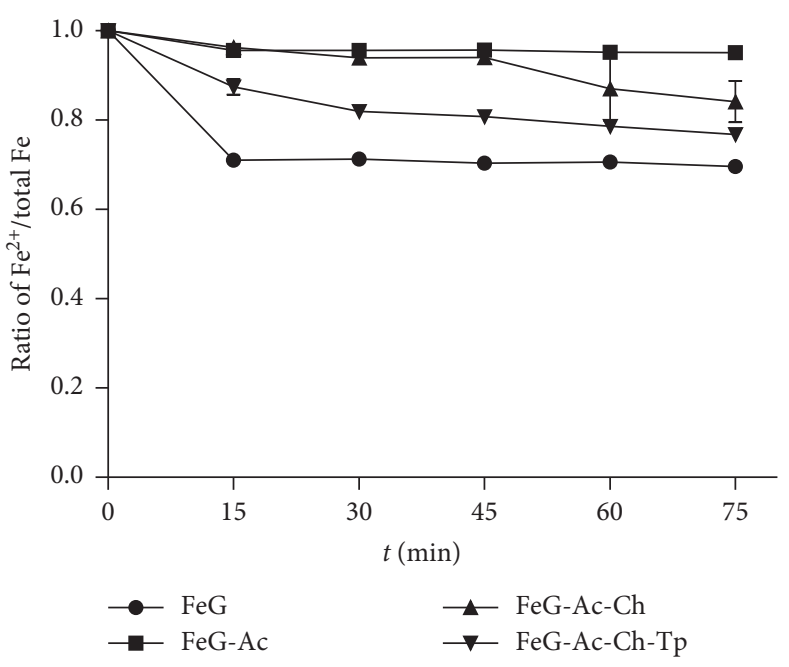

FIgURE 6: The oxidation rate of soluble ferrous iron in a batch test.

In a previous study, a kinetic equation for the oxidation of ferrous iron to the ferric iron in an aqueous solution, with $\mathrm{O}_{2}$ as the electron acceptor, was given in equation (2) [34]. This equation demonstrates that the oxidation rate of ferrous iron will decrease by twofold for every unit decrease in $\mathrm{pH}$. The presence of Ac decreased the $\mathrm{pH}$ from 4.1 to 3.3; the oxidation rate of FeG-Ac was very low in the more acidic solution (Table 2) [34].

$$
\frac{-d\left[\mathrm{Fe}^{2+}\right]}{\mathrm{d} t}=k\left[\mathrm{Fe}^{2+}\right]\left[\mathrm{OH}^{-}\right]^{2} p_{\mathrm{O}_{2}} .
$$

However, the presence of $\mathrm{Ch}$ and $\mathrm{Tp}$ increased both the $\mathrm{pH}$ value and the oxidation rate of ferrous iron, although the rate was not as high as that of the FeG solution (Table 2 and Figure 6). Figure 6 also shows that ferrous iron concentration in FeG-Ac-Ch and FeG-Ac-Ch-Tp tended to decrease in the last $60 \mathrm{~min}$ of the experiment.

A possible mechanism for the reaction that occurred during iron microparticle preparation is described in the reaction below (equations (3)-(5)). Initially, both FeG and Ac were ionised in the aqueous phase, as described in equations (3) and (4), respectively. The normal oxidation mechanism for ferrous iron is shown in equation (5). However, the higher amount of $\mathrm{H}^{+}$produced by Ac ionisation (equation (4)) would increase the amount of $\mathrm{H}^{+}$in equation (5); thus, the equilibrium would be driven towards the left side, and iron would appear in its reactive state. Furthermore, the presence of $\mathrm{Tp}$ would increase the $\mathrm{pH}$, thereby decreasing the amount of $\mathrm{H}^{+}$, and the equilibrium would shift towards the right side; thus, ferrous oxidation 
would occur (equation (5)). The feasibility of FeG and Ac ionisation were shown by the dissociation constant for gluconic acid $\left(\mathrm{Ka}=1.99 \times 10^{-4}\right)$ and $\mathrm{Ac}\left(\mathrm{Ka}=1.76 \times 10^{-5}\right)$.

$$
\begin{aligned}
\mathrm{Fe}\left(\mathrm{C}_{6} \mathrm{H}_{11} \mathrm{O}_{7}\right)_{2(\mathrm{aq})} & \Longleftrightarrow \mathrm{Fe}_{(\mathrm{aq})}^{2+}+2 \mathrm{C}_{6} \mathrm{H}_{11} \mathrm{O}_{7(\mathrm{aq})}^{-} \\
\mathrm{CH}_{3} \mathrm{COOH} & \Longleftrightarrow \mathrm{CH}_{3} \mathrm{COO}^{-}+\mathrm{H}^{+} \\
4 \mathrm{Fe}^{2+}+\mathrm{O}_{2}+10 \mathrm{H}_{2} \mathrm{O} & \Longleftrightarrow 4 \mathrm{Fe}(\mathrm{OH})_{3}+8 \mathrm{H}^{+}
\end{aligned}
$$

In practice, $\mathrm{Ch}$ - Tp microparticles can be prepared by ionic gelation, freeze drying, spray drying, etc. However, this study of $\mathrm{CV}$ and oxidation rates indicates that the presence of $\mathrm{Tp}$ increases the likelihood that ferrous iron will be transformed into ferric iron. Therefore, spray drying might be a preferable method of microparticle preparation due to the low amount of Tp (3-12\% of the total volume) in the feed solution $[36,38]$.

\section{Conclusions}

In this study, the likelihood of ferrous oxidation was investigated using $\mathrm{CV}$ and then confirmed experimentally. The results show that there was oxidation of the FeG complex solution used for preparation of $\mathrm{Ch}$ - Tp microparticles. This was evidenced by a more negative anodic potential and a decrease of ferrous concentration in the presence of $\mathrm{Ch}$ and Tp. The results demonstrate a likelihood of oxidation in the order FeG $>$ FeG-Ac-Ch-Tp $>$ FeG-Ac-Ch $>$ FeG-Ac. These findings suggest that the spray drying method is preferable to minimise the transformation of ferrous iron into ferric iron.

\section{Data Availability}

The data used to support the findings of this study are included within the article.

\section{Conflicts of Interest}

The authors declare that there are no conflicts of interest regarding the publication of this paper.

\section{Acknowledgments}

This study was funded by Universitas Indonesia through the TADOK research grant contract number NKB-0164/ UN2.R3.1/HKP.05.00/2019. The authors would also like to thank Dr. Yunita Sadeli, the Head of Corrosion Laboratory, Department of Metallurgical and Material Engineering, Universitas Indonesia, for permission to use the Autolab Metrohm Potentiostat device.

\section{References}

[1] E. Habeych, V. van Kogelenberg, L. Sagalowicz, M. Michel, and N. Galaffu, "Strategies to limit colour changes when fortifying food products with iron," Food Research International, vol. 88, pp. 122-128, 2016.

[2] M. Sari, M. W. Bloem, S. de Pee, W. J. Schultink, and S. Sastroamidjojo, "Effect of iron-fortified candies on the iron status of children aged 4-6y in East Jakarta, Indonesia," The
American Journal of Clinical Nutrition, vol. 73, no. 6, pp. 1034-1039, 2001.

[3] G. Barragán-Ibañez, A. Santoyo-Sánchez, and C. O. RamosPeñafiel, "Iron deficiency anaemia," Revista Médica del Hospital General de México, vol. 79, no. 2, pp. 88-97, 2016.

[4] K. Kalaivani, "Prevalence \& consequences of anaemia in pregnancy," Indian Journal of Medical Research, vol. 130, no. 5, pp. 627-633, 2009.

[5] L. Percy, D. Mansour, and I. Fraser, "Iron deficiency and iron deficiency anaemia in women," Best Practice \& Research Clinical Obstetrics \& Gynaecology, vol. 40, no. Supplement C, pp. 55-67, 2017.

[6] S. Akhtar, F. M. Anjum, and M. A. Anjum, "Micronutrient fortification of wheat flour: recent development and strategies," Food Research International, vol. 44, no. 3, pp. 652-659, 2011.

[7] J. S. Barkley, K. L. Kendrick, K. Codling, S. Muslimatun, and H. Pachón, "Anaemia prevalence over time in Indonesia: estimates from the 1997, 2000, and 2008 Indonesia family life surveys," Asia Pacific Journal of Clinical Nutrition, vol. 24, no. 3, pp. 452-455, 2015.

[8] C. Gupta, P. Chawla, S. Arora, S. K. Tomar, and A. K. Singh, "Iron microencapsulation with blend of gum Arabic, maltodextrin and modified starch using modified solvent evaporation method-milk fortification," Food Hydrocolloids, vol. 43, pp. 622-628, 2015.

[9] Y. Kurniawan, S. Muslimatun, E. Achadi, and S. Sastroamidjojo, "Anaemia and iron deficiency anaemia among youg adolescent girls from peri urban coastal area of Indonesia," Asia Pacific Journal of Clinical Nutrition, vol. 15, no. 3, p. 350, 2006.

[10] C. R. Titaley and M. J. Dibley, "Factors associated with not using antenatal iron/folic acid supplements in Indonesia: the 2002/2003 and 2007 Indonesia demographic and health survey," Asia Pacific Journal of Clinical Nutrition, vol. 24, no. 1, pp. 162-176, 2015.

[11] J. Berger, F. T. Wieringa, A. Lacroux, and M. A. Dijkhuizen, "Strategies to prevent iron deficiency and improve reproductive health," Nutrition Reviews, vol. 69, no. suppl_1, pp. S78-S86, 2011.

[12] L. P. McMahon, "Iron deficiency in pregnancy," Obstetric Medicine, vol. 3, no. 1, pp. 17-24, 2010.

[13] N. Abbaspour, R. Hurrell, and R. Kelishadi, "Review on iron and its importance for human health," Journal of Research in Medical Sciences: The Official Journal of Isfahan University of Medical Sciences, vol. 19, no. 2, p. 164, 2014.

[14] G. Gutiérrez, M. Matos, P. Barrero, D. Pando, O. Iglesias, and C. Pazos, "Iron-entrapped niosomes and their potential application for yogurt fortification," $L W T$, vol. 74, pp. 550-556, 2016.

[15] N. A. Handayani, M. Luthfansyah, E. Krisanti, S. Kartohardjono, and K. Mulia, "Preparation, physical characterization, and stability of ferrous-chitosan microcapsules using different iron sources," in Proceedings of the AIP Conference, AIP Publishing, Miri, Malaysia, December 2017.

[16] S. J. Kim, J. Ahn, J. S. Seok, and H. S. Kwak, "Microencapsulated iron for drink yogurt fortification," AsianAustralasian Journal of Animal Sciences, vol. 16, no. 4, pp. 581-587, 2003.

[17] N. Perez-Moral, M. C. Gonzalez, and R. Parker, "Preparation of iron-loaded alginate gel beads and their release characteristics under simulated gastrointestinal conditions," Food Hydrocolloids, vol. 31, no. 1, pp. 114-120, 2013. 
[18] A. E. Thumser, A. A. Rashed, P. A. Sharp, and J. K. Lodge, "Ascorbate enhances iron uptake into intestinal cells through formation of a $\mathrm{FeCl}_{3}$-ascorbate complex," Food Chemistry, vol. 123, no. 2, pp. 281-285, 2010.

[19] S. H. Zlotkin, C. Schauer, S. O. Agyei et al., "Demonstrating zinc and iron bioavailability from intrinsically labeled microencapsulated ferrous fumarate and zinc gluconate sprinkles in young children," The Journal of Nutrition, vol. 136, no. 4, pp. 920-925, 2006.

[20] V. Webber, D. de Siqueira Ferreira, P. L. M. Barreto, V. WeissAngeli, and R. Vanderlinde, "Preparation and characterization of microparticles of $\beta$-cyclodextrin/glutathione and chitosan/glutathione obtained by spray-drying," Food Research International, vol. 105, pp. 432-439, 2018.

[21] M. George and T. E. Abraham, "Polyionic hydrocolloids for the intestinal delivery of protein drugs: alginate and chitosan-a review," Journal of Controlled Release, vol. 114, no. 1, pp. 1-14, 2006.

[22] S.-L. Wang, Đ. M. Hiep, P. M. Luong et al., "Preparation of chitosan nanoparticles by spray drying, and their antibacterial activity," Research on Chemical Intermediates, vol. 40, no. 6 , pp. 2165-2175, 2014.

[23] V. Tokárová, O. Kašpar, Z. Knejzlík, P. Ulbrich, and F. Štěpánek, "Development of spray-dried chitosan microcarriers for nanoparticle delivery," Powder Technology, vol. 235, pp. 797-805, 2013.

[24] K. F. Babu, R. S. Kumar, M. A. Kulandainathan, and M. Noel, "Ferric-oxalate-gluconate based redox mediated electrochemical system for vat dyeing," Journal of Applied Electrochemistry, vol. 39, no. 7, pp. 1025-1031, 2009.

[25] T. Bechtold and A. Turcanu, "Fe $\mathrm{F}^{3+}$--gluconate and $\mathrm{Ca}^{2+}$ $\mathrm{Fe}^{3+}$--gluconate complexes as mediators for indirect cathodic reduction of vat dyes-cyclic voltammetry and batch electrolysis experiments," Journal of Applied Electrochemistry, vol. 34, no. 12, pp. 1221-1227, 2004.

[26] M. H. Bridge, E. Williams, M. E. G. Lyons, K. F. Tipton, and W. Linert, "Electrochemical investigation into the redox activity of $\mathrm{Fe}(\mathrm{II}) / \mathrm{Fe}(\mathrm{III})$ in the presence of nicotine and possible relations to neurodegenerative diseases," Biochimica et Biophysica Acta (BBA)-Molecular Basis of Disease, vol. 1690, no. 1, pp. 77-84, 2004.

[27] O. Firuzi, A. Lacanna, R. Petrucci, G. Marrosu, and L. Saso, "Evaluation of the antioxidant activity of flavonoids by "ferric reducing antioxidant power" assay and cyclic voltammetry," Biochimica et Biophysica Acta (BBA) - General Subjects, vol. 1721, no. 1-3, pp. 174-184, 2005.

[28] S. Mohr and T. Bechtold, "Electrochemical behaviour of ironcomplexes in presence of competitive ligands: a strategy for optimization of current density," Journal of Applied Electrochemistry, vol. 31, no. 3, pp. 363-368, 2001.

[29] E. Rudnik, "Effect of gluconate ions on electroreduction phenomena during manganese deposition on glassy carbon in acidic chloride and sulfate solutions," Journal of Electroanalytical Chemistry, vol. 741, pp. 20-31, 2015.

[30] E. Rudnik, M. Wojnicki, and G. Włoch, "Effect of gluconate addition on the electrodeposition of nickel from acidic baths," Surface and Coatings Technology, vol. 207, pp. 375-388, 2012.

[31] M. El-Khateeb, H. Abul-Futouh, H. Görls, W. Weigand, and L. R. Almazahreh, "Synthesis, characterization and electrochemical investigations of heterocyclic-selenocarboxylate iron complexes," Inorganica Chimica Acta, vol. 449, pp. 14-19, 2016.
[32] A. J. Bard, L. R. Faulkner, J. Leddy, and C. G. Zoski, Electrochemical Methods: Fundamentals and Applications, vol. 2, Wiley, New York, NY, USA, 1980.

[33] A. Jacobs and P. M. Miles, "Role of gastric secretion in iron absorption," Gut, vol. 10, no. 3, pp. 226-229, 1969.

[34] B. Morgan and O. Lahav, "The effect of $\mathrm{pH}$ on the kinetics of spontaneous $\mathrm{Fe}(\mathrm{II})$ oxidation by $\mathrm{O}_{2}$ in aqueous solution-basic principles and a simple heuristic description," Chemosphere, vol. 68, no. 11, pp. 2080-2084, 2007.

[35] B. N. Estevinho, F. Rocha, L. Santos, and A. Alves, "Microencapsulation with chitosan by spray drying for industry applications-a review," Trends in Food Science \& Technology, vol. 31, no. 2, pp. 138-155, 2013.

[36] K. G. H. Desai and H. J. Park, "Preparation and characterization of drug-loaded chitosan-tripolyphosphate microspheres by spray drying," Drug Development Research, vol. 64, no. 2, pp. 114-128, 2005.

[37] C. A. Johnson and J. A. Thomas, "The stability of aqueous solutions of ferrous gluconate," Journal of Pharmacy and Pharmacology, vol. 6, no. 1, pp. 1037-1047, 1954.

[38] K. G. H. Desai and H. J. Park, "Encapsulation of vitamin C in tripolyphosphate cross-linked chitosan microspheres by spray drying," Journal of Microencapsulation, vol. 22, no. 2, pp. 179-192, 2005. 